\title{
Robotic-assisted repair of pelvic organ prolapse: a scoping review of the literature
}

\author{
Jeffrey S. Schachar, Catherine A. Matthews
}

Female Pelvic Medicine and Reconstructive Surgery, Departments of Urology and Obstetrics and Gynecology, Wake Forest Baptist Health, Winston-Salem, NC, USA

Contributions: (I) Conception and design: All authors; (II) Administrative support: CA Matthews; (III) Provision of study materials or patients: None; (IV) Collection and assembly of data: All authors; (V) Data analysis and interpretation: All authors; (VI) Manuscript writing: All authors; (VII) Final approval of manuscript: All authors.

Correspondence to: Catherine A. Matthews, MD. Professor of Urology and Gynecology, Female Pelvic Medicine and Reconstructive Surgery, Departments of Urology and Obstetrics and Gynecology, Wake Forest Baptist Health, 1 Medical Center Boulevard, Watlington- $5^{\text {th }}$ Floor, Winston-Salem, NC 27103, USA. Email: camatthe@wakehealth.edu.

\begin{abstract}
The purpose of this article is to perform a scoping review of the medical literature regarding the efficacy, safety, and cost of robotic-assisted procedures for repair of pelvic organ prolapse in females. Sacrocolpopexy is the "gold standard" repair for apical prolapse for those who desire to maintain their sexual function, and minimally-invasive approaches offer similar efficacy with fewer risks than open techniques. The introduction of robotic technology has significantly impacted the field, converting what would have been a large number of open abdominal sacrocolpopexy (ASC) procedures to a minimally-invasive approach in the United States. Newer techniques such as nerve-sparing dissection at the sacral promontory, use of the iliopectineal ligaments and natural orifice vaginal sacrocolpopexy may improve patient outcomes. Prolapse recurrence is consistently noted in at least $10 \%$ of patients regardless of route of mesh placement. Ancillary factors including pre-operative prolapse stage, retention of the cervix, type of mesh implant, and genital hiatus (GH) size all adversely affect surgical efficacy, while trainees do not. Minimally-invasive apical repair procedures are suited to early recovery after surgery protocols but may not be appropriate for all patients. Studies evaluating longer-term outcomes of robotic sacrocolpopexies are needed to understand the relative risk/benefit ratio of this technique. With several emerging robotic platforms with improved features and a focus on decreasing costs, the future of robotics seems bright.
\end{abstract}

Keywords: Apical prolapse; minimally invasive; robot; sacrocolpopexy; pelvic organ prolapse; surgical repair; review

Submitted Aug 01, 2019. Accepted for publication Oct 04, 2019.

doi: $10.21037 /$ tau.2019.10.02

View this article at: http://dx.doi.org/10.21037/tau.2019.10.02

\section{Introduction}

Symptomatic pelvic organ prolapse has a relatively high prevalence and women have a $13-20 \%$ lifetime risk of having a surgical procedure to repair vaginal support defects $(1,2)$. Despite the anterior vaginal compartment being the most common site of pelvic organ prolapse, the loss of apical support is typically associated with more severe forms of prolapse, especially when it extends beyond the hymen. The apical compartment includes the uterus/ cervix or the vaginal cuff after a hysterectomy. Restoration of apical support is critical when repairing advanced pelvic organ prolapse, and helps to prevent failures in the other compartments $(3,4)$.

Abdominal sacrocolpopexy (ASC) has been the 'gold standard' surgery for apical support defects due to its superior outcomes and durability over native tissue vaginal repairs $(5,6)$. Despite success rates deteriorating slightly 
over time, with roughly $75 \%$ anatomic success after 7 years, sacrocolpopexy continues to outperform vaginal native tissue repairs which have a disappointing $60-70 \%$ failure rate at 5 years $(7,8)$. A recent 7 -year longitudinal study following up on 280 women who had laparoscopic sacrocolpopexy reported only a $3.3 \%$ rate of repeat surgery for recurrent pelvic organ prolapse (9).

A minimally-invasive approach to sacrocolpopexy using standard laparoscopic instruments emerged in the mid1990s and was quickly touted as superior to ASC due to shorter recovery time, reduced pain and complications (10). Laparoscopic sacrocolpopexy, however, is considered a technically challenging procedure due to the sutureintensive nature of the procedure and dissection around critical structures such as the common iliac artery and vein, factors that limited widespread adoption. Secondary to improved visualization and ergonomics with the use of wristed instruments, the introduction of robotic-assisted laparoscopic surgery has allowed more surgeons to adopt a minimally invasive approach to the sacrocolpopexy procedure (11). As such, the proportion of minimallyinvasive sacrocolpopexies performed has increased in the past decade (12). While meta-analyses have not demonstrated superior outcomes for hysterectomy or sacrocolpopexy when using the robot over standard laparoscopy, these studies fail to highlight that roboticassisted surgery has enabled the transition of many of these procedures from an open to a minimally-invasive technique (13).

Robotic-assisted prolapse repair has now been performed for over a decade with wide variability in pre-operative medical decision making and preparation, surgical technique and outcomes $(14,15)$. Our objective for this scoping review of robotic-assisted pelvic organ prolapse repair is to evaluate the comparative outcomes and to highlight the techniques with the highest efficacy and lowest rates of complications. The goal of this review is to help inform surgeons and improve pre-operative counseling, as they assist patients in a shared decision-making model to arrive at the most appropriate and individualized prolapse management for the individual patient.

\section{Learning curve}

While robotic-assisted surgery is an enabling technology, there is an initial steep learning curve where case times are initially prolonged. Several studies have demonstrated that surgical efficiency of robotic-assisted sacrocolpopexy dramatically improves after approximately 20 cases $(16,17)$. Surgical proficiency, however, may take longer. Using cumulative sum analysis, van Zanten et al. determined that 78 robotic cases were required to demonstrate a consistently low complication rate (18). Using a smaller sample from two attending surgeons, Myers et al. also demonstrated that approximately 65 cases predicted proficiency (19). These numbers are helpful targets for credentialing and maintenance of certification.

\section{Efficacy of robotic prolapse repairs}

\section{Robotic sacrocolpopexy}

Sacrocolpopexy is traditionally performed for women with post-hysterectomy vaginal vault prolapse. In women who present with uterine prolapse, the decision for concomitant hysterectomy versus uterine preservation is required. Most of the published literature on sacrocolpopexy has been collected on women with vaginal vault prolapse but this data is often extrapolated to women who present with uterine prolapse. Since laparoscopic techniques were developed prior to the robotic techniques, there is significantly more research comparing open to laparoscopic as opposed to robotic-assisted sacrocolpopexy. Two recent systematic reviews, which included over 20 comparative trials and over 5,000 patients, concluded that both techniques are equally efficacious for repairing apical vaginal prolapse, but the minimally-invasive technique is associated with fewer complications $(20,21)$. Several systematic reviews have specifically compared outcomes of laparoscopic versus robotic-assisted sacrocolpopexy and report no difference in anatomic or subjective outcomes. From these reviews, it would seem reasonable to infer that sacrocolpopexy of any type [ASC, laparoscopic sacralcolpopexy (LSC), or robotic sacralcolpopexy (RSC)] are equally efficacious (22-24). Therefore, the decision on which route is best should be determined by evaluating other factors, such as complication rates, post-operative recovery, surgeon preference, and surgical factors like uterine size or adnexal pathology.

Overall, studies evaluating outcomes of robotic-assisted apical prolapse repair report high success rates, albeit with short and medium-term follow up (Table 1). Two randomized trials comparing LSC to RSC observed no significant differences in outcomes with success rates of $88-91 \%$, and similar rates of complications $(34,35)$. One retrospective review of over 450 minimally-invasive sacrocolpopexies 
Table 1 Reported success and complication rates of robotic sacrocolpopexy

\begin{tabular}{|c|c|c|c|c|}
\hline Author & $\begin{array}{l}\text { Number of } \\
\text { patients }\end{array}$ & $\begin{array}{c}\text { Length of } \\
\text { follow-up, months }\end{array}$ & Success & Complications \\
\hline \multirow[t]{2}{*}{ Serati (25) } & 1,488 & - & Anatomical: $84-100 \%$ & Conversion to open: $<1 \%$ \\
\hline & & & & Mesh exposure: $2 \%$ \\
\hline Bradley (26) & 452 & - & - & $5.3 \times$ increased risk of failure in the group with $\mathrm{GH} \geq 4$ \\
\hline Anand (27) & 337 & 60 & - & Re-operation for prolapse: $5-8 \%$ \\
\hline van Zanten (28) & 305 & 12 & Anatomical: 65-67\% & Intra-op complications: $5 \%$ \\
\hline Gupta (29) & & & & Mesh exposure: $6.3 \%$ \\
\hline \multirow[t]{3}{*}{ Mueller (30) } & 181 & 3 & Anatomical: $85.6 \%$ & Ileus: $2.6 \%$ \\
\hline & & & & Mesh exposure: $1.1 \%$ \\
\hline & & & & Re-operation for prolapse: $0.9 \%$ \\
\hline van Zanten (31) & 166 & $>12$ & - & Mesh exposure: $1 \%$ \\
\hline \multirow[t]{2}{*}{ Hach (32) } & 101 & 22 & Subjective: $75 \%$ & Intra-op complications: $6 \%$ \\
\hline & & & & Post-op complications: $5 \%$ \\
\hline
\end{tabular}

$\mathrm{GH}$, genital hiatus; op, operation.

reported a prolapse recurrence rate of $\sim 11 \%$ with no difference between robotic versus laparoscopic routes of surgery (30). A second retrospective case series of 195 RSC identified a $7 \%$ prolapse recurrence rate, with a retreatment rate of less than $5 \%$ (29). Similarly, in systematic reviews, whether restricting to randomized trials or including nonrandomized comparative trials, the outcomes between LSC and RSC are high and do not differ significantly $(23,24)$. In a systematic review of RSC that included 27 studies with approximately 1,500 patients, subjective cure rates ranged from $92-95 \%$ and objective cure rates from $84-100 \%$ (25). One prospective cohort study of 305 women undergoing robotic-assisted sacrocolpopexy with or without concomitant supracervical hysterectomy evaluated outcomes in each vaginal compartment individually and noted that although apical anatomical success rates were over $91 \%$, all compartment anatomical success rates were much lower ( 66\%) mainly due to anterior compartment failure (28).

Currently, high quality long-term RSC outcome data does not yet exist. In a prospective case series of over 100 RSC there was a $75 \%$ subjective success rate at 22 months (32). One case series reported on 30 RSC patients after 3 years and reported a $13 \%$ rate of repeat surgery (33). In a retrospective study of over 100 RSC patients, retreatment rates were $8 \%$ at 5 years, with no difference in symptom relief (27).

\section{Ancillary factors impacting efficacy}

The surgical efficacy of sacrocolpopexy is impacted by many more important factors than route of procedure including choice of graft material, type of suture for graft attachment, concomitant procedures, pre-operative stage of prolapse, 
size of the genital hiatus $(\mathrm{GH})$ and whether the surgery is for primary or recurrent disease.

Despite robotic-assisted technology enabling the timeintensive suturing portion of the SCP procedure, it is still the portion of the operation that is most amenable to improvements in operative efficiency. Several different studies have examined different methods of graft attachment (36-38). One study compared the pull-out force of permanent versus absorbable, as well as barbed versus non-barbed sutures and found that when equivalent caliber suture was used, they all had similar strengths (36). Another study evaluating the comparative efficacy of barbed sutures reported lower satisfaction scores but greater efficiency of suturing (38). Transitioning from a permanent to an absorbable suture for vaginal graft attachment has not obviously compromised outcomes. One retrospective study reviewed the use of absorbable suture on both the vaginal and sacral ends of the mesh. They noted only a $7 \%$ rate of repeat surgery at 3-year follow-up (39).

Type 1 polypropylene mesh is the most commonly employed graft material for minimally-invasive sacrocolpopexy given the lowest risk of mesh-related complications (7,14,40-42). Ultra-lightweight polypropylene mesh materials with a large pore size that are designed to minimize the risk of mesh infection, exposure, and erosion, have been recently introduced. The lightweight materials, however, may compromise the durability of support. In a retrospective cohort study of women implanted with a heavier weight mesh $(<35 \mathrm{~g} / \mathrm{m})$ vs. an ultra-lightweight mesh $(<20 \mathrm{~g} / \mathrm{m})$, the subjects in the ultra-lightweight mesh group had twice the hazard risk of failure in the anterior compartment within 3 years of surgery. The overall rate of failure for both groups was $8 \%$. There was approximately a 5 -fold greater hazard for ultra-lightweight mesh failure in the anterior compartment in the failure group (43).

Primary versus recurrent prolapse as well as preoperative prolapse stage can have a significant impact on surgical outcomes. A retrospective review of a mesh database demonstrated that a primary repair is more successful than a repair for a recurrence. In that study, the success rates dropped to $50-60 \%$ for the same procedures when they were performed for recurrent prolapse (44). A multicenter cohort study demonstrated that a higher preoperative prolapse stage increased the risk of recurrence at 1 year by a factor of 3.8 (45). Like in cancer surgery, standard therapies are less effective when used on the more advanced and recurrent disease.

A final factor that has been recently identified that has a compromising effect on the outcomes of apical repairs is the size of the levator hiatus. In a retrospective review of over 400 patients who underwent RSC, the anatomic failure of those with an enlarged $\mathrm{GH}(\geq 4 \mathrm{~cm})$ that was not reduced at the time of surgery had a 5 -fold increased risk of anatomic failure (26).

Patients are frequently concerned about the impact of surgical trainees and the negative impact they may have on their surgery. Carter-Brooks et al., performed a retrospective review of $208 \mathrm{RSC}$ and noted that although fellows took about 30 minutes longer, there was no impact on the overall complication or recurrence rates as compared to cases without fellows (46). In addition, Crane et al., noted that although total robot docked times were longer for trainees (23 minutes), the overall average hysterectomy and sacrocolpopexy times were not different when trainees were involved in patient care (47).

In summary, regarding the existing literature on roboticassisted sacrocolpopexy for apical prolapse repair, the procedure appears highly effective for support of the vaginal apex, but does not necessarily confer the same benefit to the mid-vaginal compartments. The anterior compartment appears most vulnerable to symptomatic recurrence. This could potentially be addressed with greater anterior vaginal wall dissection, application of a dual mesh so that differential support could be provided to the anterior and posterior compartments and avoidance of mesh materials that are ultra-lightweight. In addition, consideration for concomitant perineorrhaphy in women with a wide $\mathrm{GH}$ appears essential to long-term success of robotic-assisted sacrocolpopexy.

\section{Robotic-assisted repair of uterine prolapse}

A randomized trial comparing native tissue vaginal repair to sacrocolpopexy for women with uterine prolapse has not yet been reported. If a surgeon advises a woman with uterine prolapse to undergo sacrocolpopexy, a decision with a patient-specific risk-benefit ratio is required regarding uterine preservation or removal. In the case of concomitant hysterectomy, a similar decision is required regarding cervical preservation or removal. Below we outline the relative outcomes and associated risk of each choice.

\section{Robotic-assisted hysterectomy and sacrocolpopexy}

Two studies have demonstrated longer operative time but fewer complications for women undergoing concomitant 
robotic hysterectomy and sacrocolpopexy compared to those undergoing sacrocolpopexy alone $(28,48)$. This is likely explained by the increased risk for adhesive disease and distortion of the surgical planes in women with posthysterectomy vaginal vault prolapse.

In women who elect concomitant hysterectomy, the decision for supracervical versus total hysterectomy rests on the risk-benefit ratio between mesh exposure, subsequent cervical pathology, abnormal bleeding, unanticipated uterine pathology, and prolapse outcomes. Roboticassisted total hysterectomy and sacrocolpopexy have been associated with higher rates of mesh exposure (please see complication section of paper) $(41,49,50)$ but lower rates of recurrent anterior vaginal wall prolapse. In a retrospective cohort study of 83 women undergoing robotic supracervical versus total hysterectomy and sacrocolpopexy, those in the supracervical group demonstrated a significantly higher rate of recurrent anterior compartment prolapse $(41.9 \%$ vs. $20.0 \%, \mathrm{P}=0.03$ ) (51). Similarly, in a prospective cohort study of women undergoing robotic-assisted sacrocolpopexy versus supracervical hysterectomy and cervicosacropexy, rates of recurrent anterior wall prolapse were higher in the cervical preservation group (28). One may argue that overall it would be better to remove the cervix to decrease the risk of abnormal pathology and improve prolapse reconstruction, in exchange for the higher, albeit small risk of apical mesh exposure.

\section{Hysteropexy}

Historically, uterine removal was advised as a routine component to pelvic organ prolapse repair as it seems to slightly decrease recurrence rates. However, a recent metaanalysis concluded that although there were similar anatomic outcomes and re-operation rates when hysteropexy was compared to hysterectomy, hysteropexy techniques had higher rates of subjective awareness of prolapse (52). With increased patient awareness and engagement in medical decision-making, however, there is an emerging trend towards consideration for uterine preserving techniques. Robotic-assisted hysteropexy involves the attachment of either a dual-mesh arm that traverses the broad-ligament or a single posterior mesh arm that supports the posterior vaginal wall and cervix to the anterior longitudinal ligament. In a prospective cohort study of 37 patients who completed 5-year of follow-up, the overall success rate of robotic-assisted sacral hysteropexy was $89 \%$ with significantly improved quality of life (53). In a prospective cohort study of minimally-invasive hysteropexy versus transvaginal mesh hysteropexy, women in the abdominal repair group had equivalent anatomic outcomes but improved sexual function (54).

\section{Alternatives to sacral promontory fixation}

As the sacrum has been associated with rare but significant complications of hemorrhage, discitis and ureteral injury, surgeons have evaluated alternative points of apical mesh fixation. A new modification of sacrocolpopexy termed "pectopexy" has been described in which the vaginal apex is suspended using two mesh arms to the lateral iliopectineal ligaments as opposed to the sacral promontory. This modification, first described by Banerjee and Noé in 2011, avoids the risk of vascular, ureteral and sacral disc complications that can be encountered at the promontory (55). Usta et al., published a video showing that this technique may be performed robotically as well (56). Several small studies compare pectopexy to standard laparoscopic sacrocolpopexy and ASC, and the initial findings show similar apical efficacy $(57,58)$.

Another modification for women with anterior/apical prolapse involves passage of lateral mesh arms that are cut in the shape of a " $T$ " through the lateral anterior abdominal wall where the round ligament exits the peritoneal cavitythis is termed laparoscopic lateral suspension and was originally described by Dubuisson (59). The mesh arms are not actually sutured to the anterior abdominal wall but rather are tunneled above the fascia to exit the lateral skin above the iliac crest. Retroperitoneal fibrosis results in fixation of the mesh. Giannini et al. reported the first successful case of a single-site robotic-assisted apical lateral suspension for a woman with stage III anterior/apical pelvic organ prolapse that was performed in 155 minutes (60).

\section{Mesh-less options}

Patients may choose to avoid the use of any mesh in their surgical repairs, however laparoscopic surgery may be preferred over the traditional vaginal route due to uterine size, adnexal pathology, concern for intra-peritoneal adhesions, concomitant laparoscopic procedures, or malignancy requiring surgical staging. Some native tissue procedures have been adapted for standard laparoscopy as well as robotic-assisted approaches. Several standard laparoscopic uterosacral studies have been performed with very good outcomes and low retreatment rates $(61,62)$. Robotic uterosacral ligament suspensions have 
been performed as well, although studies are limited (63). Although mesh-less alternatives are available, long-term comparative trials are needed to fully understand the efficacy of such procedures and how they compare to meshaugmented repairs.

\section{Surgical risks/complications of robotic-assisted repairs}

The abdominal approach to apical prolapse repairs has an overall low rate of complications. However, it is also associated with some serious risks, including small bowel injury/obstruction, sacral discitis, hemorrhage, and meshrelated complications. Surgeons must be aware of the presenting signs of these complications, as early diagnosis and treatment are critical. This is especially true for elderly patients who may have less medical reserve. Conflicting data regarding the relative risks of robotic sacrocolpopexy in elderly women exists. In one retrospective comparative study of women over 65 undergoing native tissue versus robotic repairs, rates of Clavien-Dindo grade III complications (those requiring surgical, endoscopic or radiologic intervention) were more common in the robotic group (48). However, in another similar study of 136 elderly women, there were no differences in outcomes or complications (64). In older women, the robotic approach should be reserved for those who are interested in preserving penetrative vaginal intercourse $(65,66)$.

Minimally-invasive sacrocolpopexy has reduced the complication rate of open ASC but thus far, no additional benefit has been afforded by the robotic approach. In a retrospective analysis of over 4,000 women using the American College of Surgeons National Surgical Quality Improvement Program Database who underwent abdominal $(\mathrm{n}=1,179) v s$. minimally-invasive sacrocolpopexy $(\mathrm{n}=3,183)$, the minimally invasive sacrocolpopexy group was associated with significantly lower rates of deep vein thrombosis, pulmonary embolism, surgical site infections, shorter hospitalization, fewer blood transfusions, 30 person-days readmission rate $(2.0 \%$ vs. $2.7 \%, \mathrm{P} \leq 0.0001)$, and 30 -day reoperation rates $(1.1 \%$ vs. $1.4 \%, \mathrm{P}<0.0001)$. In the multivariable analysis, minimally invasive sacrocolpopexy was independently associated with a reduced risk of 30-day complications [odds ratio (OR), 0.46 ; 95\% confidence interval $(\mathrm{CI}), 0.28-0.76 ; \mathrm{P}=0.002]$, blood transfusion (OR, 0.33; 95\% CI, 0.15-0.74; $\mathrm{P}=0.007$ ), prolonged hospitalization (OR, 0.16; 95\% CI, 0.12-0.23; $\mathrm{P}<0.001$ ), and readmission [hazard ratio (HR), 0.62; 95\%
CI, 0.41-0.96; $\mathrm{P}=0.03]$ (10).

Systematic reviews comparing laparoscopic and roboticassisted sacrocolpopexy concluded that there was no difference between the two techniques with regards to complication rates $(22,24)$. In a retrospective review of RSC, intra-operative complications were found to be low ( $2 \%$ cystotomy, $2 \%$ vaginotomy, $1 \%$ conversion to open) (29). RSC has also been associated with lower estimated blood loss and shorter hospitalization, but a higher rate of intraoperative complications as compared to ASC (67).

Small bowel injury and obstruction occur more commonly after sacrocolpopexy than native tissue vaginal repair. A systematic review comparing outcomes of mesh sacrocolpopexy to native tissue vaginal repairs reported that rates of ileus and small bowel obstruction were approximately 3 -fold higher in the sacrocolpopexy group (5). In a retrospective review of 450 minimally-invasive sacrocolpopexy procedures, in which $86 \%$ did not undergo retro-peritonealization of mesh, they found that $3 \%$ of women had small bowel complications, and half required surgical management (30). In general, with robotic surgery, it is imperative to be aware of the entire surgical field especially when using monopolar energy which has the potential to arc to tissues not directly in the field of view.

Bowel injury during initial laparoscopic entry is another risk of minimally invasive surgery (68). Rates of small bowel injury may be under-appreciated in our literature as prospective randomized trials are not designed to capture rare events. In a retrospective evaluation of 144 studies that included over 13,000 women who underwent roboticassisted gynecologic surgery reported that $1 / 160$ women were diagnosed with bowel injury (69). In a review of 475,000 gynecologic procedures, 350 bowel injuries were diagnosed and $41 \%$ of them were categorized as delayed recognition (68). Surgeons must perform a careful physical exam and inspect the abdominal wall for any evidence of surgical scars on the skin that might predict a higher risk of bowel adhesions to the anterior abdominal wall. Aggressive assessment of post-operative nausea and vomiting with an oral contrast abdominal computer-assisted tomography imaging study is recommended to investigate for small bowel injury. Plain abdominal X-rays do not provide enough information in the post-operative setting. Surgeons should investigate for visceral injury with the use of imaging with oral contrast in someone who appears to have an ileus after laparoscopic or robotic surgery.

Mesh exposure is a unique risk of sacrocolpopexy that has been mitigated by an overall reduction in mesh weight and increase in mesh pore size over time. The extended 
CARE trial, in which about $40 \%$ of implants were not type 1 polypropylene mesh, there was a $10.5 \%$ risk of mesh exposure noted up to 7 years after surgery (7). Similarly, in a study comparing RSC with either robotic total laparoscopic hysterectomy (TLH) or supracervical hysterectomy, 14\% of patients in the TLH group had mesh exposure and none were noted in the other group. However, $4.4 \%$ of the supracervical hysterectomy group were subsequently found to have abnormal pathology (41). More recent studies have observed mesh exposure in a range from about $1 \%$ to $6 \%$, however with shorter follow up $(29,31,70)$. Although mesh exposure rates may increase over time, the adverse impact on patient quality of life may not be as devastating as previously thought and can frequently be managed in a conservative fashion. Despite the risk of mesh exposure, the benefits of a superior longer-lasting surgical outcome may outweigh the relatively high rate of recurrence associated with native tissue repairs.

Dyspareunia and pelvic pain are commonly reported adverse events in most studies of pelvic floor surgery. The risk of these complications is lower with sacrocolpopexy as compared to native tissue vaginal repairs (6). Sexual satisfaction has been shown to improve with sacrocolpopexy. A prospective study assessed sexual satisfaction of both partners, and after RSC, both men and women had higher sexual satisfaction scores (71). Reviews of RSC concluded that RSC has more post-operative pain than $\operatorname{LSC}(22,23)$. Interestingly, when bupivacaine was injected into the incision sites during a RSC, there was no significant improvement in pain scores as compared to saline (72). When RSC was compared to vaginal native tissue repair, the RSC group used fewer narcotic equivalents although there were no differences in pain scales (73). Post-operative pain is a risk associated with any surgery. Ensuring that the light-weight mesh is placed in the proper surgical plane without considerable tension is critical to decrease rates of pain after sacrocolpopexy.

Ureteral injury is more common after vaginal native tissue repairs, such as uterosacral ligament suspension, than sacrocolpopexy. Given that these are relatively rare events, there are limited published data on ureteral injury during robotic sacrocolpopexy specifically. Regardless, cystoscopy confirming ureteral patency is indicated to ensure an intact genitourinary system.

Post-operative constipation occurs in $10-50 \%$ of sacrocolpopexy and is likely related to the interruption of the hypogastric plexus at the sacral promontory. The pectopexy technique, which avoids this plexus by not attaching the mesh to the sacrum, has been associated with lower rates of obstructed defecation (57). A nervesparing LSC technique has been used recently which was inspired by cadaveric dissection that preserves the superior hypogastric plexus, the right hypogastric nerve, the lumbosacral sympathetic trunk and the inferior hypogastric plexus (74). While additional research is needed on this technique, early reports highlight the importance of understanding the relevant anatomy and that preserving crucial structures is important. Similar techniques may be used robotically as well to decrease post-operative constipation.

De novo stress urinary incontinence (SUI) is always a concern when correcting apical and anterior prolapse. In the CARE trial, the estimated probability of developing de novo SUI after ASC was 0.77 in the control group (7). A retrospective study of sacrocolpopexies found that $45 \%$ of patients undergoing ASC had de novo SUI vs. $15 \%$ in the minimally-invasive sacrocolpopexy group. In addition, they found that de novo SUI was associated with a greater change of point "Aa" on the POP-Q (75). Davenport et al., came to a similar conclusion when performing a secondary analysis of the CARE trial, that the incidence of de novo SUI was directly correlated to the degree of anterior compartment prolapse on the pre-operative examination (76). Proper tensioning of the mesh is important to adequately correct the vaginal prolapse, but surgeons should be cautioned against "over-tensioning" the mesh, which may increase the risk of de novo SUI. When performing robotic-assisted sacrocolpopexy, it may be necessary for the surgeon to leave the surgical console to perform a vaginal examination and ensure appropriate tension.

As with standard laparoscopy, there have been significantly more reports of patients discharged home on the day of surgery. Kisby et al., retrospectively compared 80 same-day discharges to 192 subjects discharged at least 24 hours after RSC. They found no significant differences between the groups in terms of unplanned provider visits, clinic visits, emergency room visits, readmissions, or number of phone calls to the office (77). While same-day discharge is probably not feasible for all patients, it may be a reasonable option for the appropriate patient.

As surgeons continue to gain experience and more data is published on techniques, the rate of complications is declining. In a recent review of adverse events reported to the FDA MAUDE database, there was a peak of adverse events in 2014 (124 events) which then dropped to 7 events in 2016. They noted that over a 10 -year period, $88.6 \%$ 
of the reported events were due to robotic malfunctions, $9.9 \%$ due to injury, and $1.5 \%$ due to death. They also found that when malfunctions occurred it rarely led to a serious injury or impacted surgical approach (78). This data however should be viewed with the understanding that the MAUDE database is a passive database and requires people to proactively report complications which means many complications may not be reported.

\section{Cost}

Healthcare costs continue to be a concern throughout the world. New technological advances in minimally invasive surgery have improved patient outcomes with shorter hospital stay and quicker recovery but come at a price. When the initial cost of purchasing and maintenance of the robot is included, RSC has significantly higher costs than LSC (34,35). Surgical efficiency was the principal driver of the higher cost findings in the Paraiso trial. Geller and Matthews also demonstrated that sequential reductions in robotic case times resulted in cost reductions and overall programmatic profitability (16). This was similarly noted in other studies where the higher RSC costs were mostly attributed to longer operative times resulting in higher anesthetic costs $(29,79)$. In order to combat these longer times, instituting a dedicated robotic team has been shown to decrease operating times by 26 minutes (80).

Interestingly, in a study comparing costs of vaginal apical prolapse reconstruction versus sacrocolpopexy, vaginal reconstruction was significantly less expensive than both ASC and RSC which were similarly expensive (68). In contrast, Patel et al., found minimally-invasive sacrocolpopexies were more expensive than ASC (81). Treatment of recurrent prolapse should also factor into cost models. While the initial investment is clearly higher for mesh sacrocolpopexy than native tissue repair, lower rates of re-intervention may balance out the cost equation.

\section{Developing trends}

Several new techniques have been studied in RSC to improve patient outcomes. Single-port RSC, which can aide in cosmesis, has been reported as feasible by several surgeons (82-84). In addition to improving cosmesis, a single-port RSC may facilitate quicker uterine corpus removal and decreases the risk of port-site infection given that there are fewer ports (82). Using barbed suture to attach the mesh vaginally, as well as pulling the mesh to the sacrum via a retroperitoneal tunnel created using the wristed instruments are recommended by the authors to improve surgical proficiency $(83,84)$. In addition, Yaghnam et al., were able to perform a robotic natural orifice surgery through the vagina for pelvic organ prolapse repair on two cadavers (85). While some of these techniques may be viable options, more research is needed, and they are not widely used.

\section{Conclusions}

Sacrocolpopexy is the "gold standard" repair for apical prolapse for those who desire to maintain their sexual function, and minimally-invasive approaches offer similar efficacy with fewer risks than open techniques. The introduction of robotic technology has significantly impacted the field, converting what would have been a large number of open ASC procedures to a minimally-invasive approach in the United States. Newer techniques such as nerve-sparing dissection at the sacral promontory, use of the iliopectineal ligaments and natural orifice vaginal sacrocolpopexy may improve patient outcomes. Prolapse recurrence is consistently noted in at least $10 \%$ of patients regardless of route of mesh placement. Ancillary factors including preoperative prolapse stage, retention of the cervix, type of mesh implant, and GH size all adversely affect surgical efficacy, while trainees do not. Minimally-invasive apical repair procedures are suited to early recovery after surgery protocols but may not be appropriate for all patients. Studies evaluating longer-term outcomes of robotic sacrocolpopexies are needed to understand the relative risk/benefit ratio of this technique. With several emerging robotic platforms with improved features and a focus on decreasing costs, the future of robotics seems bright.

\section{Acknowledgments}

Funding: None.

\section{Footnote}

Provenance and Peer Review: This article was commissioned by the Guest Editor (Ashok K. Hemal) for the series "Robotic-assisted Urologic Surgery" published in Translational Andrology and Urology. The article was sent for external peer review organized by the Guest Editor and the editorial office.

Conflicts of Interest: All authors have completed the ICMJE 
uniform disclosure form (available at http://dx.doi. org/10.21037/tau.2019.10.02). The series "Roboticassisted Urologic Surgery" was commissioned by the editorial office without any funding or sponsorship. CAM: Boston Scientific-grant support and consultant; Johnson \& Johnson-expert witness. JSS has no other conflicts of interest to declare.

Ethical Statement: The authors are accountable for all aspects of the work in ensuring that questions related to the accuracy or integrity of any part of the work are appropriately investigated and resolved.

Open Access Statement: This is an Open Access article distributed in accordance with the Creative Commons Attribution-NonCommercial-NoDerivs 4.0 International License (CC BY-NC-ND 4.0), which permits the noncommercial replication and distribution of the article with the strict proviso that no changes or edits are made and the original work is properly cited (including links to both the formal publication through the relevant DOI and the license). See: https://creativecommons.org/licenses/by-nc$\mathrm{nd} / 4.0 /$.

\section{References}

1. Wu JM, Matthews CA, Conover MM, et al. Lifetime risk of stress urinary incontinence or pelvic organ prolapse surgery. Obstet Gynecol 2014;123:1201-6.

2. Smith FJ, Holman CD, Moorin RE, et al. Lifetime risk of undergoing surgery for pelvic organ prolapse. Obstet Gynecol 2010;116:1096-100.

3. Brubaker L, Maher C, Jacquetin B, et al. Surgery for pelvic organ prolapse. Female Pelvic Med Reconstr Surg 2010;16:9-19.

4. Hsu Y, Chen L, Summers A, et al. Anterior vaginal wall length and degree of anterior compartment prolapse seen on dynamic MRI. Int Urogynecol J Pelvic Floor Dysfunct 2008;19:137-42.

5. Siddiqui NY, Grimes CL, Casiano ER, et al. Mesh sacrocolpopexy compared with native tissue vaginal repair. Obstet Gynecol 2015;125:44-55.

6. Maher C, Feiner B, Baessler K, et al. Surgery for women with apical vaginal prolapse. Cochrane Database Syst Rev 2016;10:CD012376.

7. Nygaard I, Brubaker L, Zyczynski HM, et al. Long-term outcomes following abdominal sacrocolpopexy for pelvic organ prolapse. JAMA 2013;309:2016-24.
8. Jelovsek JE, Barber MD, Brubaker L, et al. Effect of uterosacral ligament suspension vs sacrospinous ligament fixation with or without perioperative behavioral therapy for pelvic organ vaginal prolapse on surgical outcomes and prolapse symptoms at 5 years in the OPTIMAL randomized clinical trial. JAMA 2018;319:1554-65.

9. Pacquée S, Nawapun K, Claerhout F, et al. Longterm assessment of a prospective cohort of patients undergoing laparoscopic sacrocolpopexy. Obstet Gynecol 2019;134:323-32.

10. Linder BJ, Occhino JA, Habermann EB, et al. A national contemporary analysis of perioperative outcomes of open versus minimally invasive sacrocolpopexy. J Urol 2018;200:862-7.

11. Carroll AW, Lamb E, Hill AJ, et al. Surgical management of apical pelvic support defects: the impact of robotic technology. Int Urogynecol J 2012;23:1183-6.

12. Slopnick EA, Petrikovets A, Sheyn D, et al. Surgical trends and patient factors associated with the treatment of apical pelvic organ prolapse from a national sample. Int Urogynecol J 2019;30:603-9.

13. Lawrie TA, Liu H, Lu D, et al. Robot-assisted surgery in gynaecology. Cochrane Database Syst Rev 2019;4:CD011422.

14. O'Sullivan OE, Matthews CA, O'Reilly BA. Sacrocolpopexy: is there a consistent surgical technique? Int Urogynecol J 2016;27:747-50.

15. Takacs EB, Kreder KJ. Sacrocolpopexy: surgical technique, outcomes, and complications. Curr Urol Rep 2016;17:90.

16. Geller EJ, Lin FC, Matthews CA. Analysis of robotic performance times to improve operative efficiency. $\mathrm{J}$ Minim Invasive Gynecol 2013;20:43-8.

17. Akl MN, Long JB, Giles DL, et al. Robotic-assisted sacrocolpopexy: technique and learning curve. Surg Endosc 2009;23:2390-4.

18. van Zanten F, Schraffordt Koops SE, Pasker-De Jong PCM, et al. Learning curve of robot-assisted laparoscopic sacrocolpo(recto)pexy: a cumulative sum analysis. Am J Obstet Gynecol 2019;221:483.e1-483.e11.

19. Myers EM, Geller EJ, Connolly A, et al. Robotic sacrocolpopexy performance and cumulative summation analysis. Female Pelvic Med Reconstr Surg 2014;20:83-6.

20. De Gouveia De Sa M, Claydon LS, Whitlow B, et al. Laparoscopic versus open sacrocolpopexy for treatment of prolapse of the apical segment of the vagina: a systematic review and meta-analysis. Int Urogynecol J 2016;27:3-17.

21. Coolen AWM, Bui BN, Dietz V, et al. The treatment of post-hysterectomy vaginal vault prolapse: a 
systematic review and meta-analysis. Int Urogynecol J 2017;28:1767-83.

22. Callewaert G, Bosteels J, Housmans S, et al. Laparoscopic versus robotic-assisted sacrocolpopexy for pelvic organ prolapse: a systematic review. Gynecol Surg 2016;13:115-23.

23. De Gouveia De Sa M, Claydon LS, Whitlow B, et al. Robotic versus laparoscopic sacrocolpopexy for treatment of prolapse of the apical segment of the vagina: a systematic review and meta-analysis. Int Urogynecol J 2016;27:355-66.

24. Pan K, Zhang Y, Wang Y, et al. A systematic review and meta-analysis of conventional laparoscopic sacrocolpopexy versus robot-assisted laparoscopic sacrocolpopexy. Int J Gynaecol Obstet 2016;132:284-91.

25. Serati M, Bogani G, Sorice P, et al. Robot-assisted sacrocolpopexy for pelvic organ prolapse: a systematic review and meta-analysis of comparative studies. Eur Urol 2014;66:303-18.

26. Bradley MS, Askew AL, Vaughan MH, et al. Roboticassisted sacrocolpopexy: early postoperative outcomes after surgical reduction of enlarged genital hiatus. Am J Obstet Gynecol 2018;218:514.e1-8.

27. Anand M, Weaver AL, Fruth KM, et al. Symptom relief and retreatment after vaginal, open, or robotic surgery for apical vaginal prolapse. Female Pelvic Med Reconstr Surg 2017;23:297-309.

28. van Zanten F, Schraffordt Koops SE, O’Sullivan OE, et al. Robot-assisted surgery for the management of apical prolapse: a bi-centre prospective cohort study. BJOG 2019;126:1065-73.

29. Gupta P, Ehlert M, Bartley J, et al. Perioperative outcomes, complications, and efficacy of robotic-assisted prolapse repair: a single institution study of 196 patients. Female Pelvic Med Reconstr Surg 2018;24:408-11.

30. Mueller MG, Jacobs KM, Mueller ER, et al. Outcomes in 450 women after minimally invasive abdominal sacrocolpopexy for pelvic organ prolapse. Female Pelvic Med Reconstr Surg 2016;22:267-71.

31. van Zanten F, van Iersel JJ, Hartog FE, et al. Mesh exposure after robot-assisted laparoscopic pelvic floor surgery: a prospective cohort study. J Minim Invasive Gynecol 2019;26:636-42.

32. Hach CE, Krude J, Reitz A, et al. Midterm results of robot-assisted sacrocolpopexy. Int Urogynecol J 2015;26:1321-6.

33. Jong K, Klein T, Zimmern PE. Long-term outcomes of robotic mesh sacrocolpopexy. J Robot Surg
2018;12:455-60.

34. Anger JT, Mueller ER, Tarnay C, et al. Robotic compared with laparoscopic sacrocolpopexy. Obstet Gynecol 2014;123:5-12.

35. Paraiso MFR, Jelovsek JE, Frick A, et al. Laparoscopic compared with robotic sacrocolpopexy for vaginal prolapse. Obstet Gynecol 2011;118:1005-13.

36. Pilkinton ML, Levine GC, Bennett L, et al. Comparison of strength of sacrocolpopexy mesh attachment using barbed and nonbarbed sutures. Int Urogynecol J 2018;29:153-9.

37. Stubbs JT. Short-term results of robotic sacrocolpopexy using the Quill SRS bi-directional polydioxanone (PDO) suture. J Robot Surg 2011;5:259-65.

38. Tan-Kim J, Nager CW, Grimes CL, et al. A randomized trial of vaginal mesh attachment techniques for minimally invasive sacrocolpopexy. Int Urogynecol J 2015;26:649-56.

39. Linder BJ, Anand M, Klingele CJ, et al. Outcomes of robotic sacrocolpopexy using only absorbable suture for mesh fixation. Female Pelvic Med Reconstr Surg 2017;23:13-6.

40. Warembourg S, Labaki M, de Tayrac R, et al. Reoperations for mesh-related complications after pelvic organ prolapse repair: 8 -year experience at a tertiary referral center. Int Urogynecol J 2017;28:1139-51.

41. Osmundsen BC, Clark A, Goldsmith C, et al. Mesh erosion in robotic sacrocolpopexy. Female Pelvic Med Reconstr Surg 2012;18:86-8.

42. Moroni RM, Juliato CRT, Cosson M, et al. Does sacrocolpopexy present heterogeneity in its surgical technique? A systematic review. Neurourol Urodyn 2018;37:2335-45.

43. Askew AL, Visco AG, Weidner AC, et al. Does mesh weight affect time to failure after robotic-assisted laparoscopic sacrocolpopexy? Female Pelvic Med Reconstr Surg 2018. [Epub ahead of print].

44. Trochez RD, Lane S, Duckett J, et al. The use of synthetic mesh for vaginal prolapse in the UK: a review of cases submitted to the British Society of Urogynaecology database. Int Urogynecol J 2018;29:899-904.

45. Aslam MF, Osmundsen B, Edwards SR, et al. Preoperative prolapse stage as predictor of failure of sacrocolpopexy. Female Pelvic Med Reconstr Surg 2016;22:156-60.

46. Carter-Brooks CM, Du AL, Bonidie MJ, et al. The impact of fellowship surgical training on operative time and patient morbidity during robotics-assisted sacrocolpopexy. Int Urogynecol J 2018;29:1317-23.

47. Crane AK, Geller EJ, Matthews CA. Trainee performance 
at robotic console and benchmark operative times. Int Urogynecol J 2013;24:1893-7.

48. Dubinskaya A, Hernandez-Aranda D, Wakefield DB, et al. Comparing laparoscopic and robotic sacrocolpopexy surgical outcomes with prior versus concomitant hysterectomy. Int Urogynecol J 2020;31:401-7.

49. Warner WB, Vora S, Hurtado EA, et al. Effect of operative technique on mesh exposure in laparoscopic sacrocolpopexy. Female Pelvic Med Reconstr Surg 2012;18:113-7.

50. Crane AK, Geller EJ, Sullivan S, et al. Short-term mesh exposure after robotic sacrocolpopexy with and without concomitant hysterectomy. South Med J 2014;107:603-6.

51. Myers EM, Siff L, Osmundsen B, et al. Differences in recurrent prolapse at 1 year after total vs supracervical hysterectomy and robotic sacrocolpopexy. Int Urogynecol J 2015;26:585-9.

52. Meriwether KV, Antosh DD, Olivera CK, et al. Uterine preservation vs hysterectomy in pelvic organ prolapse surgery: a systematic review with meta-analysis and clinical practice guidelines. Am J Obstet Gynecol 2018;219:129-46.e2.

53. Grimminck K, Mourik SL, Tjin-Asjoe F, et al. Longterm follow-up and quality of life after robot assisted sacrohysteropexy. Eur J Obstet Gynecol Reprod Biol 2016;206:27-31.

54. Gutman RE, Rardin CR, Sokol ER, et al. Vaginal and laparoscopic mesh hysteropexy for uterovaginal prolapse: a parallel cohort study. Am J Obstet Gynecol 2017;216:38.e1-11.

55. Banerjee C, Noé KG. Laparoscopic pectopexy: a new technique of prolapse surgery for obese patients. Arch Gynecol Obstet 2011;284:631-5.

56. Usta T, Karacan T, Kale A, et al. Robot-assisted laparoscopic pectouteropexy: an alternative uterussparing technique for pelvic organ prolapse surgery. Int Urogynecol J 2017;28:1751-3.

57. Noé KG, Schiermeier S, Alkatout I, et al. Laparoscopic pectopexy: a prospective, randomized, comparative clinical trial of standard laparoscopic sacral colpocervicopexy with the new laparoscopic pectopexy-postoperative results and intermediate-term follow-up in a pilot study. J Endourol 2015;29:210-5.

58. Biler A, Ertas IE, Tosun G, et al. Perioperative complications and short-term outcomes of abdominal sacrocolpopexy, laparoscopic sacrocolpopexy, and laparoscopic pectopexy for apical prolapse. Int Braz J Urol 2018;44:996-1004.
59. Dubuisson JB, Yaron M, Wenger JM, et al. Treatment of genital prolapse by laparoscopic lateral suspension using mesh: a series of 73 patients. J Minim Invasive Gynecol 2008;15:49-55.

60. Giannini A, Russo E, Mannella P, et al. Single site roboticassisted apical lateral suspension (SS R-ALS) for advanced pelvic organ prolapse: first case reported. J Robot Surg 2017;11:259-62.

61. Haj Yahya R, Chill HH, Herzberg S, et al. Anatomical outcome and patient satisfaction after laparoscopic uterosacral ligament hysteropexy for anterior and apical prolapse. Female Pelvic Med Reconstr Surg 2018;24:352-5.

62. Houlihan S, Kim-Fine S, Birch C, et al. Uterosacral vault suspension (USLS) at the time of hysterectomy: laparoscopic versus vaginal approach. Int Urogynecol J 2019;30:611-21.

63. Davila HH, Gallo T, Bruce L, et al. Robotic and laparoendoscopic single-site utero-sacral ligament suspension for apical vaginal prolapse: evaluation of our technique and perioperative outcomes. J Robot Surg 2017;11:171-7.

64. Robinson BL, Parnell BA, Sandbulte JT, et al. Robotic versus vaginal urogynecologic surgery. Female Pelvic Med Reconstr Surg 2013;19:230-7.

65. Turner LC, Kantartzis K, Lowder JL, et al. The effect of age on complications in women undergoing minimally invasive sacral colpopexy. Int Urogynecol J 2014;25:1251-6.

66. Narins H, Danforth TL. Management of pelvic organ prolapse in the elderly - is there a role for robotic-assisted sacrocolpopexy? Robot Surg 2016;3:65-73.

67. Anand M, Weaver AL, Fruth KM, et al. Perioperative complications and cost of vaginal, open abdominal, and robotic surgery for apical vaginal vault prolapse. Female Pelvic Med Reconstr Surg 2017;23:27-35.

68. Llarena NC, Shah AB, Milad MP. Bowel injury in gynecologic laparoscopy. Obstet Gynecol 2015;125:1407-17.

69. Picerno T, Sloan NL, Escobar P, et al. Bowel injury in robotic gynecologic surgery: risk factors and management options. A systematic review. Am J Obstet Gynecol 2017;216:10-26.

70. Dandolu V, Akiyama M, Allenback G, et al. Mesh complications and failure rates after transvaginal mesh repair compared with abdominal or laparoscopic sacrocolpopexy and to native tissue repair in treating apical prolapse. Int Urogynecol J 2017;28:215-22. 
71. Culligan PJ, Haughey S, Lewis C, et al. Sexual satisfaction changes reported by men after their partners' roboticassisted laparoscopic sacrocolpopexies. Female Pelvic Med Reconstr Surg 2019;25:365-8.

72. Yeung J, Crisp CC, Mazloomdoost D, et al. Liposomal bupivacaine during robotic colpopexy and posterior repair: a randomized controlled trial. Obstet Gynecol 2018;131:39-46.

73. Westermann LB, Crisp CC, Mazloomdoost D, et al. Comparative perioperative pain and recovery in women undergoing vaginal reconstruction versus robotic sacrocolpopexy. Female Pelvic Med Reconstr Surg 2017;23:95-100.

74. Ercoli A, Cosma S, Riboni F, et al. Laparoscopic nervepreserving sacropexy. J Minim Invasive Gynecol 2017;24:1075-7.

75. LeClaire EL, Mukati MS, Juarez D, et al. Is de novo stress incontinence after sacrocolpopexy related to anatomical changes and surgical approach? Int Urogynecol J 2014;25:1201-6.

76. Davenport MT, Sokol ER, Comiter CV, et al. Does the degree of cystocele predict de novo stress urinary incontinence after prolapse repair? Further analysis of the colpopexy and urinary reduction efforts trial. Female Pelvic Med Reconstr Surg 2018;24:292-4.

77. Kisby CK, Polin MR, Visco AG, et al. Same-day discharge after robotic-assisted sacrocolpopexy. Female Pelvic Med Reconstr Surg 2019;25:337-41.

Cite this article as: Schachar JS, Matthews CA. Roboticassisted repair of pelvic organ prolapse: a scoping review of the literature. Transl Androl Urol 2020;9(2):959-970. doi: $10.21037 /$ tau.2019.10.02
78. Souders C, Nik-Ahd F, Zhao H, et al. Robotic sacrocolpopexy: adverse events reported to the FDA over the last decade. Int Urogynecol J 2019;30:1919-23.

79. Cheon C, Maher C. Economics of pelvic organ prolapse surgery. Int Urogynecol J 2013;24:1873-6.

80. Carter-Brooks CM, Du AL, Bonidie MJ, et al. The impact of a dedicated robotic team on robotic-assisted sacrocolpopexy outcomes. Female Pelvic Med Reconstr Surg 2018;24:13-6.

81. Patel M, O'Sullivan D, Tulikangas PK. A comparison of costs for abdominal, laparoscopic, and robot-assisted sacral colpopexy. Int Urogynecol J Pelvic Floor Dysfunct 2009;20:223-8.

82. Lowenstein L, Matanes E, Burke YZ. Surgical technique for robot-assisted sacrocolpopexy performed via a single port. Urology 2017;103:272.

83. Matanes E, Lauterbach R, Mustafa-Mikhail S, et al. Single port robotic assisted sacrocolpopexy. Female Pelvic Med Reconstr Surg 2017;23:e14-8.

84. Guan X, Ma Y, Gisseman J, et al. Robotic single-site sacrocolpopexy using barbed suture anchoring and peritoneal tunneling technique: tips and tricks. J Minim Invasive Gynecol 2017;24:12-3.

85. Yaghnam I, Thomas D, Rosenblatt P, et al. The feasibility of transvaginal robotic surgery in the repair of pelvic organ prolapse. Int Urogynecol J 2017;28:1263-4. 